\title{
Sample Study Population
}

National Cancer Institute

\section{Source}

National Cancer Institute. Sample Study Population. NCI Thesaurus. Code C70837.

The population from which study subjects are recruited. 